Effect of gas flow on the grouth of In-rich Al I nN fi I ms by net al-or gani c chemical vapor deposition

\begin{tabular}{|l|l|}
\hline 著者 & $\begin{array}{l}\text { 康 亭亭, 山本 政智, 田中 幹康, 橋本 明弘, 山本 } \\
\text { ?勇 }\end{array}$ \\
\hline 著者 (英) & $\begin{array}{l}\text { KANG Ti ng- Ti ng, YANAMOTO Nasat ond, TANAKA } \\
\text { M ki yasu, HASH MTO Aki hi ro, YANAMOTO Aki o }\end{array}$ \\
\hline $\begin{array}{l}\text { j our nal or } \\
\text { publ i cat i on ti t l e }\end{array}$ & Journal of Appl i ed Physi cs \\
\hline vol une & 106 \\
\hline page range & 053525- 1- 053525- 4 \\
\hline year & 2009- 08 \\
\hline URL & ht t p: //hdl . handl e. net /10098/2162 \\
\hline
\end{tabular}




\title{
Effect of gas flow on the growth of In-rich AllnN films by metal-organic chemical vapor deposition
}

\author{
Ting-Ting Kang (康亭亭), ${ }^{\text {a) }}$ Masatomo Yamamoto (山本政智), \\ Mikiyasu Tanaka (田中幹康), Akihiro Hashimoto (橋本明弘), and \\ Akio Yamamoto (山本高勇) \\ Department of Electrical and Electronics Engineering, Graduate School of Engineering, \\ University of Fukui, Bunkyo 3-9-1, Fukui 910-8507, Japan
}

(Received 6 April 2009; accepted 30 July 2009; published online 14 September 2009)

\begin{abstract}
Indium-rich AlInN are grown by metal-organic (MO) chemical vapor deposition using trimethylaluminum, trimethylindium, and ammonia. Under the conservation of MO influx, the effects of gas flow in the MO route on AlInN growth and Al-related parasitic reaction are investigated. With an increase in this gas flow, the suppression of Al-related parasitic reaction, i.e., enhancement in Al content incorporation and improvement of crystalline quality, is satisfactorily shown until the occurring of severe phase separation. Accordingly, $\mathrm{Al}$ content $x$ in $\mathrm{Al}_{x} \mathrm{In}_{1-x} \mathrm{~N}$ can be tuned from $x=0.02$ to 0.26 . The Raman spectra of those AlInN samples with phase separation are analyzed by the resonant excitation effect and two-mode behavior for $A_{1}(\mathrm{LO})$. Finally, we propose a phase diagram to interpret the phase separation and Al content evolution under the influence of gas flow. (C) 2009 American Institute of Physics. [doi:10.1063/1.3212969]
\end{abstract}

\section{INTRODUCTION}

AlInN is one hopeful semiconductor alloy for solar cell because of its wide band-gap coverage of 0.7-6.2 eV. However, the metal-organic chemical vapor deposition (MOCVD) growth of AlInN is challenging because $\mathrm{Al}$ precursor is so reactive that it readily leads to the uncontrolled parasitic reaction and incorporation of carbon and oxygen into the solid. This is particularly a problem when using trimethylaluminum (TMAl) as MO precursors, a dimmer in the gas phase, which pyrolyzes to form aluminum carbide. Even though, due to the extremely low vapor pressures of those less reactive $\mathrm{Al}$ precursor triethylaluminum and triisobutylaluminum, TMAl is still the most conveniently and widely used Al precursor. ${ }^{1}$

Previously, Koide et $a{ }^{2} .^{2}$ and Zhao et $a .^{3}$ had studied TMAl-related parasitic reaction in the MOCVD growth of $\mathrm{AlGaN}$ and AlN respectively. According to their studies, the parasitic reaction will prohibit $\mathrm{Al}$ content incorporation and degenerate the material quality. For AlInN, ${ }^{4}$ especially Inrich AlInN, ${ }^{5-7}$ which is a rising semiconductor requiring special growth conditions, e.g., low growth temperature and relatively high growth pressure, its MOCVD growth is much less studied yet. In this work, using MOCVD with TMAl, trimethylindium (TMIn), and ammonia $\left(\mathrm{NH}_{3}\right)$, we report the effect of gas flow on the growth of In-rich AlInN. The major purpose here is to grow high quality metastable In-rich AlInN by the kinetically controlled technique, aiming at controlling the Al related parasite reactions and overcoming the large thermodynamic immiscibility gap of AlInN ternary alloy.

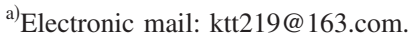

\section{EXPERIMENTS}

A horizontal reactor is used in our MOCVD system. For our system, the total carrier gas flow rate in MO line ahead of the reactor entrance is $F_{\text {bubble }}+F_{\text {main }}: F_{\text {bubble }}$ is the nitrogen gas passing through the MO source bubblers; $F_{\text {main }}$ is another route (denoted as main route) nitrogen gas used as forced flow, which plays the dominant role in hydrodynamics and mass transport. This dominance is ensured by the fact that $F_{\text {main }}$ is usually 3-8 times larger than $F_{\text {bubble }}$ during the growth.

The growth procedure is given below. After growing a thin AlN buffer on $c$-plane sapphire, AlInN growth is started under the pressure of 730 Torr at $600{ }^{\circ} \mathrm{C}$. Before this work, we have optimized the growth conditions for AlInN, such as V/III ratio, AlN buffer growth time, and AlInN growth temperature. These efforts make it possible to grow single-crystalline $\mathrm{Al}_{x} \mathrm{In}_{1-x} \mathrm{~N}(x \sim 0.15)$ film without phase separation for $F_{\text {main }}=1$ SLM (liters per minute at standard temperature and pressure). In this study, only $F_{\text {main }}$ is adjusted, while all other parameters, e.g., the influx of MO precursors $\left(F_{\text {bubble }}\right)$ and $\mathrm{NH}_{3}$, are unchanged.

\section{RESULTS}

\section{A. Surface morphology dependence on gas flow}

To give the readers a direct impression of Al-related parasitic reaction, we first study the dependence of AlInN surface morphology on the substrate placement position and gas flow. Three substrates are placed on three positions inside of the reactor. All these positions are in the direction of gas flow, with the separation from the entrance for MO precursors being 92 (position 1), 118 (position 2), and $144 \mathrm{~mm}$ (position 3).

Some representative scanning electron microscopy (SEM) images of AlInN samples are given in Fig. 1, whose 


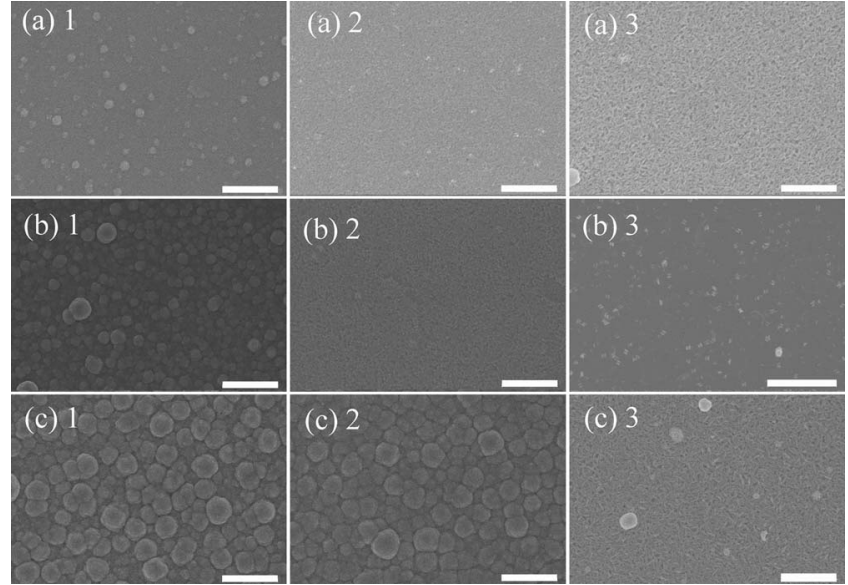

FIG. 1. SEM images of AlInN samples under different flow rate in the main route $F_{\text {main }}$ : (a) 0.5 , (b) 1 , and (c) 1.4 SLM. The characters 1,2 , and 3 mean the substrate positions 92,118 , and $144 \mathrm{~mm}$ from MO entrance. The length of white scale bar is $5 \mu \mathrm{m}$.

$F_{\text {main }}$ values are $0.5,1$, and $1.4 \mathrm{SLM}$, respectively. There is a notable dependence of morphology on the substrate positions. In the positions close to the MO entrance [position 1, Figs. 1(a)-1(c)], some particlelike microstructures appear on all the samples. These microstructures fade away when the substrate leaves the MO entrance. The density and individual size of the particles is approximately proportional to the gas flow rate $F_{\text {main }}$. So the particlelike structures prevail under a considerately large gas flow $F_{\text {main }}=1.4$ SLM in position 1 . Compared with $\mathrm{InN}$ grown by the same buffer and growth conditions, the particlelike microstructures, which disappear in case of InN growth, should be resulted from the TMAl influx.

It is known that the parasitic reaction of TMAl and $\mathrm{NH}_{3}$ occurring in the vapor phase is much more serious than that of TMIn and $\mathrm{NH}_{3}{ }^{8}$ As a result, $\mathrm{Al}$ atom has a very short mean free path, leading to the three-dimensional nuclear growth pattern of AlInN, i.e., particlelike structures. This viewpoint is also in agreement with other observations. For example, usually in positions 2 and 3, particlelike microstructure is rarely observed because of the fast exhausting of excessive $\mathrm{Al}$ precursor. Therefore it is reasonable to believe that if $F_{\text {main }}$ is large enough to reduce the TMAl contact time with $\mathrm{NH}_{3}$, the fast exhausting can be suppressed. As we expected, for a strong gas flow $F_{\text {main }}=1.4$ SLM in Fig. 1 [(c) 2], the particlelike microstructures can also be observed in position 2.

\section{B. Phase separation evolution}

Because of the uncontrolled reaction of TMAl and $\mathrm{NH}_{3}$, the samples obtained in positions 1 and 2 are usually poor in quality and weak in x-ray diffraction (XRD) intensity. Therefore, the following discussions will be limited to the films grown in position 3. Figure 2 gives the XRD spectra of the AlInN samples obtained in position 3. In Fig. 2, phase separation is both observed for $F_{\text {main }}$ above and below $F_{\text {optimal }}$. In addition to the dominant composition-In-rich InAlN $\left(\theta \sim 32.0^{\circ}\right)$, phase separation produces Al-rich AlInN, InN, and metallic indium. The evolution of the $\mathrm{Al}$ content $x$ in

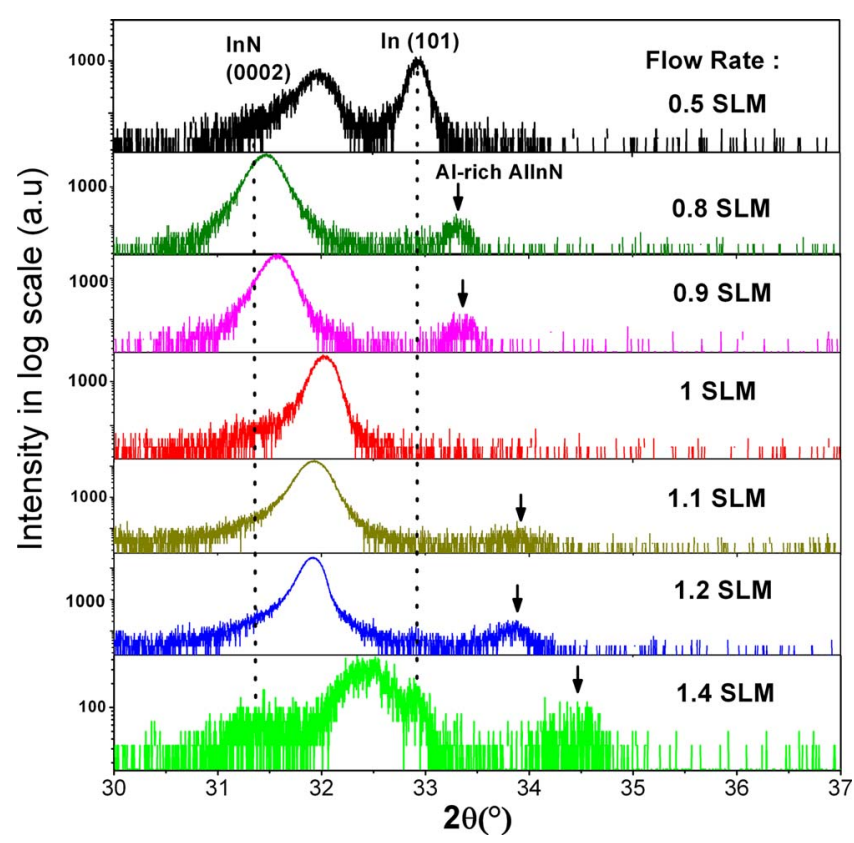

FIG. 2. (Color online) XRD spectra of AlInN grown in position 3 with different gas flow rate in main route $\left(F_{\text {main }}\right) . F_{\text {main }}$ values are given inset. The Al-rich AlInN produced by phase separation is indicated by arrows.

In-rich AlInN are depicted in Fig. 3(a).The most important phenomena in Fig. 2 is the enhancement in $\mathrm{Al}$ incorporation with an increase in $F_{\text {main }}$.

For $F_{\text {main }}=F_{\text {optimal }}=1 \mathrm{SLM}, \mathrm{Al}_{x} \mathrm{In}_{1-x} \mathrm{~N}$ with $x \sim 0.15(\theta$ $\sim 32.0^{\circ}$ ) is obtained without other phase. When $F_{\text {main }}$ increases, in addition to the main peak with $x \sim 0.15$, a new maximum from the Al-rich AlInN phase produced by phase separation appears in high $\theta$ side. This maximum shifts continually to larger $\theta$ with the increased $F_{\text {main }}$, which indicates an increase in $\mathrm{Al}$ content in the separated Al-rich AlInN phase. For $F_{\text {main }}=1.4 \mathrm{SLM}$, another separated phase-InN is also observed. The simultaneous appearance of In-rich AlInN, InN, and Al-rich AlInN means that the phase separation type here is corresponding to partial phase separation (binodal decomposition), not complete phase separation (spinodal decomposition). When $F_{\text {main }}$ decreases below

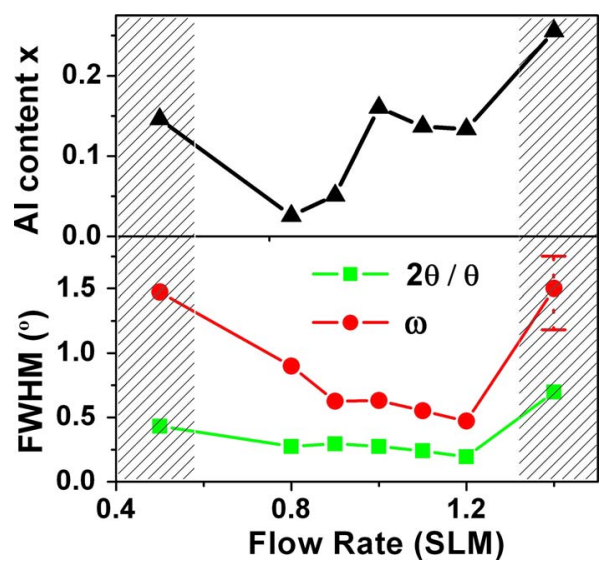

FIG. 3. (Color online) For the major composition, i.e., In-rich AlInN with $\theta \sim 32.0^{\circ}$ : (a) (above) Al content $x$ obtained by Vegard's law. (b) (below) FWHM in (0002) $2 \theta / \theta$ and $\omega$ scan as a function of $F_{\text {main }}$. The shade regions are the phase separation zones. 
$F_{\text {optimal, }}$, phase separation happens again and $\mathrm{Al}$ content in In-rich, Al-rich AlInN phase both decrease, indicating the beginning of In-rich environment. When $F_{\text {main }}=0.5$ SLM, phase separation due to In-rich environment is so serious that metallic indium appears. Because indium droplets act as sinks for In atoms and prevent the incorporation of higher indium fractions in the film, ${ }^{9-11} \mathrm{Al}$ content in In-rich AlInN increases and $\theta$ value for the maximum returns back to $\sim 32.0^{\circ}$. We have noticed the different separated phases, i.e., $\mathrm{InN} / \mathrm{metallic}$ indium under large/small gas flow. This difference implies some different phase separation mechanisms and we will discuss this issue later.

Figure 3(a) gives the evidence that the $\mathrm{Al}$ content $x$ in In-rich AlInN is enhanced by an increased $F_{\text {main }}$. This relationship is relatively well observed, except the pronounced phase separation case $F_{\text {main }}=0.5$ SLM and some small fluctuations around $F_{\text {main }}=F_{\text {optimal }}=1$ SLM. In Fig. 3(b), from $F_{\text {main }}=0.5$ to $1.2 \mathrm{SLM}$, the full width at half maximum (FWHM) both in (0002) $\theta / 2 \theta$ and $\omega$ scan of In-rich AlInN decrease continually. It should be noted that this crystal quality improvement is obtained through the suppression of parasitic reaction, not at the price of less $\mathrm{Al}$ content incorporation. Therefore it is opposite to the general trend of poorer quality for more $\mathrm{Al}$ incorporation in In-rich AlInN ${ }^{5,6}$ For $F_{\text {main }}=1.2 \mathrm{SLM}$, FWHM both in (0002) $\omega$ scan reaches $0.47^{\circ}(x=0.14)$. This is much smaller than the value reported by our group before with $x \sim 0.15 .^{5}$ The density of screw threading dislocations $\left(N_{\text {screw }}\right)$ is then estimated through the tilt angle $\beta_{\text {tilt }}=0.47^{\circ}=0.00822 \mathrm{rad}$ using the classical formula $^{12}$

$$
N_{\text {screw }}=\beta_{\text {tilt }} / 4.35 b_{c}^{2},
$$

where $b_{c}$ is the Burgers vector of $c$-type threading dislocations $\left(b_{c}=c_{\mathrm{AlInN}}=0.5604 \mathrm{~nm}\right) . N_{\text {screw }}$ for this film is estimated to be $\sim 5 \times 10^{9} \mathrm{~cm}^{-2}$.

\section{Raman scattering}

Figure 4 is the Raman spectra of some AlInN samples. Raman spectra are measured on the AlInN surface in a backscattering configuration at room temperature with $514 \mathrm{~nm}$ laser excitation. Before this paper, we have given a strong experimental support to the two-mode behavior for $A_{1}(\mathrm{LO})$ phonon in AlInN, ${ }^{13}$ which is in contrast with previously widely adopted one-mode $A_{1}(\mathrm{LO})$ phonon behavior. ${ }^{14}$ Using the linear relation of mode frequency versus $\mathrm{Al}$ content $x$ in Ref. 13

$$
\begin{aligned}
& \omega_{E_{2}^{H}}(x)=477.625+149.61 x \\
& \omega_{A_{1}(\mathrm{LO}), \text { InN-like }}(x)=581.55+138.40 x \\
& \omega_{A_{1}(\mathrm{LO}), \text { AlN-like }}(x)=619.23+257.67 x
\end{aligned}
$$

the obvious maxima in Fig. 4 are respectively identified as $E_{2}^{H}$, InN-like $A_{1}(\mathrm{LO})$ and AlN-like $A_{1}(\mathrm{LO})$ from In-rich AlInN with $\theta \sim 32.0^{\circ}$, as shown in Fig. 4. This identification is also supported by comparing the Raman spectra shapes here and those in Ref. 13.

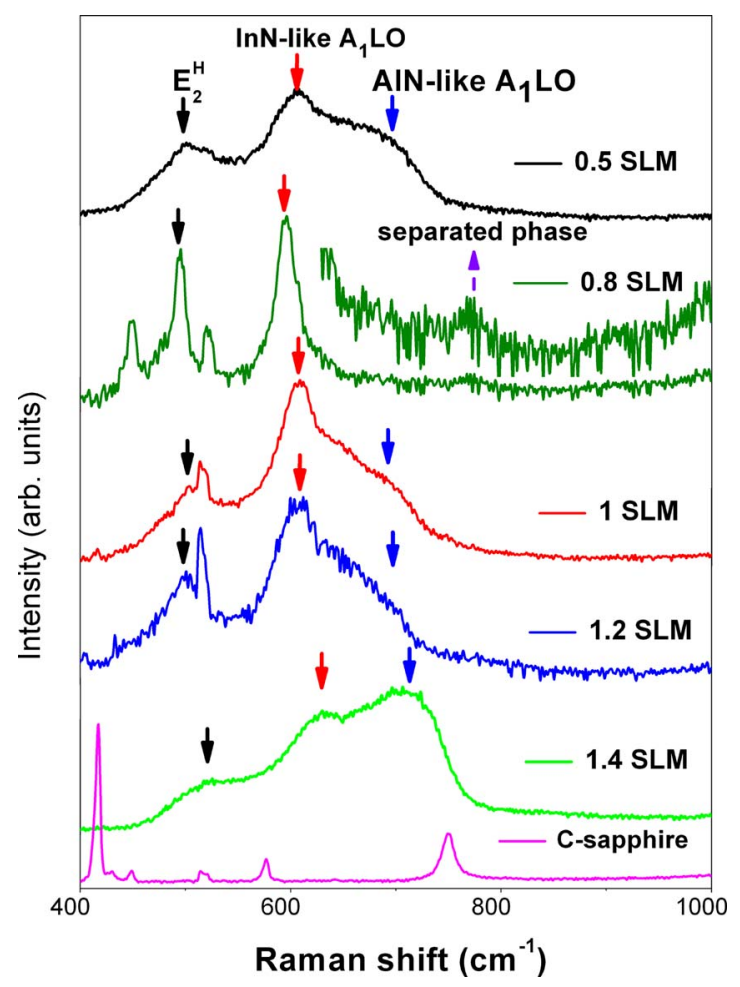

FIG. 4. (Color online) Raman spectra of AlInN grown in position 3 with different gas flow rate in main route $\left(F_{\text {main }}\right)$. The spectra in $630-1000 \mathrm{~cm}^{-1}$ for $F_{\text {main }}=0.8 \mathrm{SLM}$ sample is magnified and displayed inset.

The signals from the separated phase-Al-rich AlInN are badly observed. Only for the spectra $F_{\text {main }}=0.8$ SLM, a very weak maximum is observed around $770 \mathrm{~cm}^{-1}$. Considering the limited accuracy of Eq. (4), this maximum $\sim 770 \mathrm{~cm}^{-1}$ is believed to be from AlN-like $A_{1}(\mathrm{LO})$ of the separated phase $\mathrm{Al}_{0.47} \mathrm{In}_{0.53} \mathrm{~N}\left(\theta \sim 33.3^{\circ}\right.$ in XRD spectra).

Since the excitation laser energy $(2.41 \mathrm{eV})$ used here is near the band gap of $\mathrm{Al}_{x} \mathrm{In}_{1-x} \mathrm{~N}$ with $x \sim 0.55,{ }^{6}$ the resonant excitation effect ${ }^{14}$ is responsible for this enhanced observation of separated phase $\mathrm{Al}_{0.47} \mathrm{In}_{0.53} \mathrm{~N}$ Raman signals. Concerning the sample with $F_{\text {main }}=1.4 \mathrm{SLM}$, although the amount of Al-rich AlInN ( $\mathrm{Al}$ content $x=0.71$ deduced from Fig. 3) is relatively comparable to In-rich AlInN, their band gap is much larger than the excitation photon energy, and greatly damps their Raman signal intensities. For other samples with $F_{\text {main }}$ different from 0.8 and 1.4 SLM, the separated Al-rich AlInN phase has either too large a band gap or too small an amount, making their Raman signals invisible.

\section{DISCUSSION}

In Fig. 5, we adopt a phase diagram to explain the influence of gas flow on In-rich AlInN growth. Two factors, i.e., the adsorption/desorption of In species and the reaction time for MO precursor with $\mathrm{NH}_{3}$, are highlighted in defining this phase diagram. For $\mathrm{Al}$ parasitic reaction, a high gas flow rate will reduce the chance for $\mathrm{TMAl}$ and $\mathrm{NH}_{3}$ to come in contact and suppress the parasitic reaction. Another way to reduce the TMAl- $\mathrm{NH}_{3}$ contact chance and resultant parasitic reaction is using low reactor pressure, which has been shown by Zhao et al. ${ }^{3}$ Since the small AlInN grain formation, which contributes to dislocations density, is suppressed along with 


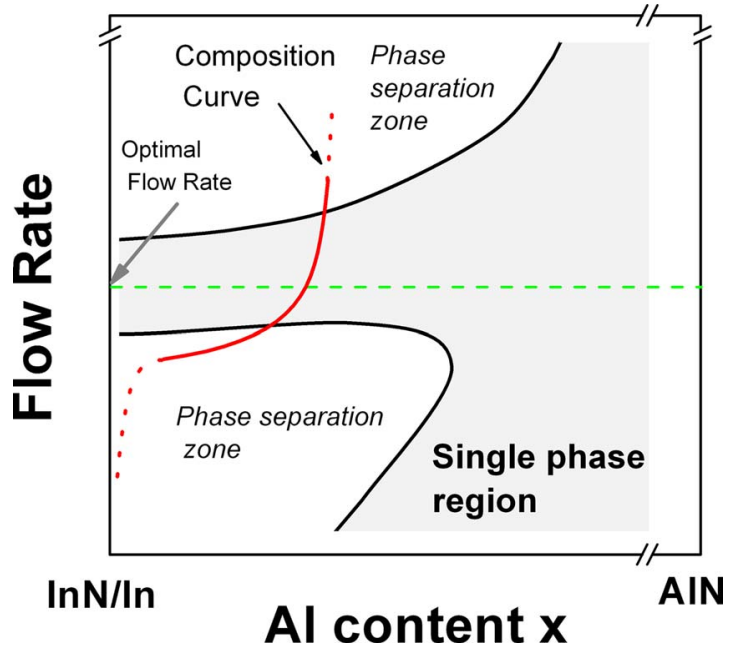

FIG. 5. (Color online) Phase diagram concerning $F_{\text {main }}$ and Al composition. The parts of composition curve deep in phase separation zones are plotted in dotted curve.

parasitic reaction, the crystalline quality is steadily improved. On the other hand, a high gas flow rate has some negative influences. At the same time of reducing the TMAl- $\mathrm{NH}_{3}$ contact chance, it also reduces the chance for the three species-In, Al, and $\mathrm{N}$ - contacting together to form AlInN. This leads to In-N and Al-N bonds forming separately, as evidenced by $\mathrm{InN}$ and $\mathrm{Al}$-rich AlInN presented in Fig. 2 at $F_{\text {main }}=1.4$ SLM. Accordingly, the crystalline quality improvement under large $F_{\text {main }}$ will be ultimately terminated by this phase separation.

Another factor, i.e., adsorption of In species, becomes more important in a slow gas flow. It is known that $\mathrm{InN}$ and metallic indium have a large equilibrium vapor pressure, ${ }^{15}$ making In species adsorption /desorption to sample surface very sensitive to the gas flow. When $F_{\text {main }}<F_{\text {optimal }}$, the subtle equilibrium between adsorption and desorption of In species to sample surface is broken. The In species adsorption will surpass desorption significantly and make phase separation inevitable. The situation will be deteriorated further when considering strong $\mathrm{Al}$ parasitic reaction under slow gas flow.

In combination of the two factors, the phase diagram in Fig. 5 is characteristic of two phase separation zones below and above the optimal gas flow rate $F_{\text {optimal }}$. During the increase in $F_{\text {main }}$, the resultant material composition is described by the composition curve. In the vicinity of phase separation zone, the composition curve experience partial phase separation, where In-rich AlInN can sustain phase separation, until the complete decomposition of In-rich AlInN by complete phase separation. One achievement of Fig. 5 is that the abnormal enhancement in $\mathrm{Al}$ content in case of $F_{\text {main }}=0.5 \mathrm{SLM}$, can be satisfactorily interpreted as the complete decomposition of unstable In-AlInN into metallic indium and more Al-rich AlInN at $\theta \sim 32.0^{\circ}$.

\section{SUMMARY}

In summary, for MOCVD growth of In-rich AlInN, the suppression of TMAl related parasitic reaction by a strong gas flow are directly observed through the enhancement in Al content incorporation, improvement of crystalline quality, and the dependence of AlInN surface morphology on substrate placement position. Although phase separation is induced in case of inappropriate gas flow, it is found that the separated Al-rich AlInN phase is almost invisible in Raman scattering due to its low amount and lack of resonant excitation effect. Two factors, the adsorption/desorption of In species and the reaction time for $\mathrm{MO}$ precursor with $\mathrm{NH}_{3}$, are found to be important for In-rich AlInN growth. The mixed influences of these two factors require an optimal gas flow configuration to grow high crystalline quality In-rich AlInN without phase separation.

\section{ACKNOWLEDGMENTS}

This work was supported by the Ministry of Education, Culture, Sports, Science and Technology of Japan through Grant-in-Aid for Scientific Research in Priority Areas (Grant No. 18069005).

${ }^{1}$ G. B. Stringfellow, Organometallic Vapor-phase Epitaxy: Theory and Practice, 2nd ed. (Academic, San Diego, 1999).

${ }^{2}$ Y. Koide, H. Itoh, N. Sawaki, I. Akasaki, and M. Hashimoto, J. Electrochem. Soc. 133, 1956 (1986).

${ }^{3}$ D. G. Zhao, J. J. Zhu, D. S. Jiang, and J. W. Liang, H. Yang, X.Y. Li, and H. M. Gong, J. Cryst. Growth 289, 72 (2006).

${ }^{4}$ S. Senda, H. Jiang, and T. Egawa, Appl. Phys. Lett. 92, 203507 (2008).

${ }^{5}$ Y. Houchin, A. Hashimoto, and A. Yamamoto, Phys. Status Solidi C 5, 1571 (2008).

${ }^{6} \mathrm{~W}$. Terashima and S.-B. Che, Y. Ishitani, and A. Yoshikawa, Jpn. J. Appl. Phys., Part 2 45, L539 (2006)

${ }^{7}$ C. Hums, J. Bläsing, A. Dadgar, A. Diez, T. Hempel, J. Christen, A. Krost, K. Lorenz, and E. Alves, Appl. Phys. Lett. 90, 022105 (2007).

${ }^{8}$ J. R. Creighton, G. T. Wang, W. G. Breiland, and M. E. Coltrin, J. Cryst. Growth 261, 204 (2004).

${ }^{9}$ D. Doppalapudi, S. N. Basu, K. F. Ludwig, Jr., and T. D. Moustakas, J. Appl. Phys. 84, 1389 (1998)

${ }^{10}$ T. Matsuoka, T. Sakai, and A. Katsui, Optoelectron., Devices Technol. 5, 53 (1990).

${ }^{11}$ E. L. Piner, F. G. McIntosh, J. C. Roberts, K. S. Boutros, M. E. Aumer, V. A. Joshkin, N. A. El-Masry, S. M. Bedair, and S. X. Liu, Mater. Res. Soc. Symp. Proc. 449, 85 (1997).

${ }^{12}$ B. N. Pantha, R. Dahal, M. L. Nakarmi, N. Nepal, J. Li, J. Y. Lin, and H. X. Jiang, Appl. Phys. Lett. 90, 241101 (2007).

${ }^{13}$ T.-T. Kang, A. Hashimoto, and A. Yamamoto, Phys. Rev. B 79, 033301 (2009).

${ }^{14}$ V. M. Naik, W. H. Weber, D. Uy, D. Haddad, R. Naik, Y. V. Danylyuk, M. J. Lukitsch, G. W. Auner, and L. Rimai, Appl. Phys. Lett. 79, 2019 (2001).

${ }^{15}$ O. Ambacher, M. S. Brandt, R. Dimitrov, T. Metzger, M. Stutzmann, R. A. Fischer, A. Miehr, A. Bergmajer, and G. Dollinger, J. Vac. Sci. Technol. B 14, 3532 (1996). 